\title{
ASSESSMENT OF THE MORPHOLOGICAL DEVELOPMENT OF THE CAECAL TONSIL IN TURKEY (Meleagris gallopavo)
}

\author{
${ }^{*}$ NNADOZIE, OKECHUKWU1; ${ }^{1}$ IKPEGBU, EKELE; ${ }^{1}$ NLEBEDUM, UCHENNA CALLISTUS; ${ }^{1}$ AGBAKWURU, ISAIAH. \\ ${ }^{1}$ Department of Veterinary Anatomy, Michael Okpara University of Agriculture, Umudike, Abia State, \\ Nigeria. \\ *correspondence to Ekele Ikpegbu drdozie15@gmail.com, Mobile : +2347085987809
}

\begin{abstract}
The post hatch $(\mathrm{PH})$ development of the caecal tonsil in turkey (Meleagris gallopavo) was studied from day (D) 1 post-hatch to D 140. The tonsils appeared as nodular structure on the surface of the proximal part of the caecal base. The caecal wall of all ages was composed of four histological layers. The mucosa consisted of simple columnar epithelium with goblet cells. At D $1 \mathrm{PH}$ the tonsil was composed of minute aggregates of proliferating lymphoid cells. By D 7, Leiberkuhn glands were observed in the stroma of the tonsil, and at D 28, nodules packed with lymphocytes and some plasma cells were observed. Between D 28 and D 133, there were gradual increase in dimension of the tonsils and proliferation of lymphatic nodules. At D 140, there was depletion of lymphatic nodules especially at the apical regions bordering the caecal lumen, and increase in thickness of the intervening connective tissue was very obvious.

Key words: caecal tonsil, turkey (Meleagris gallopavo), morphology, post-hatch, development
\end{abstract}

\section{INTRODUCTION}

The ability of organisms to resist disease is a fundamental responsibility of a defense mechanism called the immune or lymphoid system. This system is composed of several lines of defense to prevent pathogen entry and subsequent infection (Corton et al., 1998; Erf, $2004,2007)$. The avian lymphoid system is structurally divided into two distinct components namely, the primary or central and the secondary or peripheral components (Firth, 1977). The primary or central component comprises the thymus and the bursa of Fabricius in which the lymphocytes develop (Ratcliffe, 1989; Ciriaco et al., 2003). The secondary or peripheral component which apparently depends on the primary organs for their development and function includes all areas of normal lymphocytic aggregation or proliferation outside the central component. This includes the spleen and all mucosa associated lymphoid tissues (MALT) such as the caecal tonsil (Firth, 1977; Fred et al., 2008; Islam, 2012).

In galliformes, the large intestine consists of paired caeca and a short straight rectum joined to ileum and cloaca. The right and left caeca are elongated blind sacs, each consisting of proximal part or base, a long middle part or body and a short distal end or apex which extends to a pointed end (Rezaian and Hamedi, 2007). Caecum has important role in liquid absorption and cellulose digestion, but it also acts as a defensive organ due to the presence of large masses of diffuse and nodular lymphatic tissue in the lamina propria and submucosa that formed the caecal tonsils (Getty, 1975; Rezaian and Hamedi, 2007). Often the caecal tonsils are located in the proximal one third of the paired tubular caecum as ampullaceous structures on the surface of the caecum as observed in the chicken (Akter et al., 2006), though variations in location of the tonsils within the caecum have been recorded in some other studied avian species (Kitamura et al., 1976).

The morphology of the caecal tonsil has been reported in a wide range of birds, but information on its post-hatch development especially in turkey remains scanty. Therefore, the purpose of this work is to study step by step the morphological changes in the caecal 


\section{MATERIALS AND METHOD}

One hundred apparently healthy day-old turkey poults of either sex were purchased from Green Hands Agro-Vet Farms, Umuahia, Abia State, Nigeria. The birds were kept all through the experimental period in a deep-liter pen of the poultry unit of the College of Veterinary Medicine, Michael Okpara University of Agriculture, Umudike, Abia State, Nigeria; and were fed commercially compounded feed $\left(\right.$ Topfeed $\left.^{(R)}\right)$ and water was provided ad libitum. No medication of any kind including vaccination was given throughout the study period. The birds were divided into twenty groups of five birds per group comprising day $1,7,14,21,28$, $35,42,49,56,63,70,77,84,91,98,112,119$, 126,133 and 140 . Based on this schedule, five randomly selected birds were euthanized by inhalation anaesthesia using chloroform after their live weights had been determined using a weighing balance. The caeca were collected by ventral abdominal dissection (Alboghobeish and Mayahi, 2003), and examined for gross features.Slices from the base of the caecum of different ages were fixed in Bouin's fluid and transfered to $70 \%$ alcohol after 24 hours. The specimens were processed routinely for light microscopy. The tissues were embedded in paraffin wax, trimmed and mounted on wooden chuck, and then taken to the microtome for sectioning at $5 \mu \mathrm{m}$ thickness. The sections were floated in floating-out bath from where it was picked with clean albuminized slides. The slides were placed in a staining dish and excess wax was removed by two changes of xylene and hydrated by descending grades of alcohol. The slides were taken to water and then stained by filtered Ehrlich hematoxylin, washed in water, differentiated in $1 \%$ acid alcohol and blued in running tap water. It was then counter stained with filtered eosine, and excess eosine was removed in ascending grades of alcohol. It was then cleared in two changes of xylene and cover slipped with Depex mountant. The slides were viewed under a light microscope and selected images were captured using moticam.0 digital camera attached to a computer.

\section{RESULT}

The caecal tonsils were predominantly located on the proximal part or base of the paired tubular caeca. Its appearance as thick elevations on the surface of the caecal base became more pronounced with age.

Histologically, at day 1 post hatch, the four layers of the caecal wall had apparently differentiated. The mucosa was composed of simple columnar epithelium with secretory goblet cells interspersed among the columnar cells. This epithelium was thrown into high longitudinal folds of intestinal villi whose crypts communicated with developing Leiberkuhn glands. In the lamina propria-submucosa were accumulations of lymphoid cells that sat on the muscularis and lined by the epithelium (Fig. 1). These lymphoid aggregates were composed of large heterochromatic lymphocytes that formed the earliest lymphoid accumulations of the caecal tonsil. The muscularis consisted of layers of circularly oriented smooth muscle fibers.

By day 7 , there was remarkable increase in appearance of the caecal tonsil due to an increase in density of the lymphoid accumulations. Leiberkuhn glands had as well developed within the substance of the tonsil (Fig. 2). At day 14, there was further proliferation of the lymphoid cells and the associated Leiberkuhn glands had increased in number.

By day 28 , the tonsil appeared massive in nature and many spherical lymphatic nodules were observed. The Leiberkuhn glands had also increased further in number, and the intervening connective tissue had compartmentalized the tonsils (Fig. 3). Some nodules were in direct contact with the muscular layer of the caecal wall, but walled off 
from surrounding tissues by fibroblasts. The lymphatic nodules were densely populated with lymphocyte among which were plasma cells and macrophages.

By day 42, the intervening connective tissue had traversed to a great extent the substance of the tonsil. The nodules and the extra nodular regions remained densely populated with lymphocytes, while in the nodules were increased number of plasma cells.

By day 56, the tonsils had grown extensively in size and comprised many lymphatic nodules some of which were subepithelial in position (Fig. 4). The intervening connective tissues septa and the lymphatic nodular capsule had apparently thickened.

At day 84, the tonsil remained massive, and almost occluded the lumen of the caecum. The epithelium remained intact and still composed of simple columnar epithelium with goblet cells (Fig. 5). Within the tonsil was abundance of lymphatic nodules separated from one another by a network of bundles of fibrous intervening connective tissue.

By day 112, the tonsil consisted basically of lymphatic nodules that were separated from one another by thick fibrous internodular

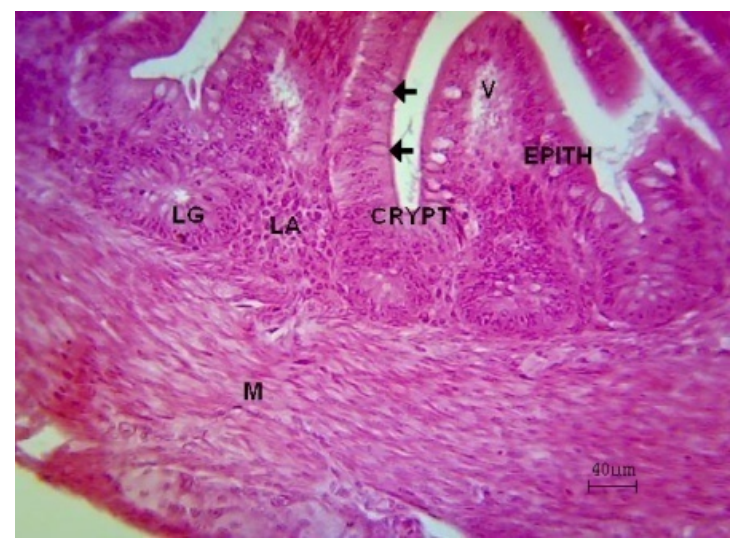

FIGURE 2. Transverse Section of the caecal tonsil at day 7 post hatch showing the dimension of the tonsils, T. Note the Leiberkuhn glands at the center of the tonsils. X100. connective tissue that harbored the Leiberkuhn glands (Fig. 6). Large cavities were observed in the axial septae, and some nodules aligned along the margins of the cavities. The size and cellular density of the nodules varied considerably, but there was still obvious high concentration of cells in each of the nodules.

At day 126, the muscularis had remarkably thickened and composed layers of bundles of smooth muscle. The interaction between the lymphatic nodule and the muscular layer of the caecal wall became prominent. The nodules were separated from the smooth muscle fibers by tuft of connective tissue while the surrounding nodular capsule remained intact (Fig. 7).

At day 140, the tonsil was still extensive in appearance, but comprised nodules that showed reduced cellular densities. There were obvious wide intercellular spaces among cells within the nodules. The nodules at the apical regions toward the caecal lumen tended to disintegrate and the internodular spaces initially occupied by diffused accumulations of lymphocytes were dominated by intervening connective tissue (Fig. 8).

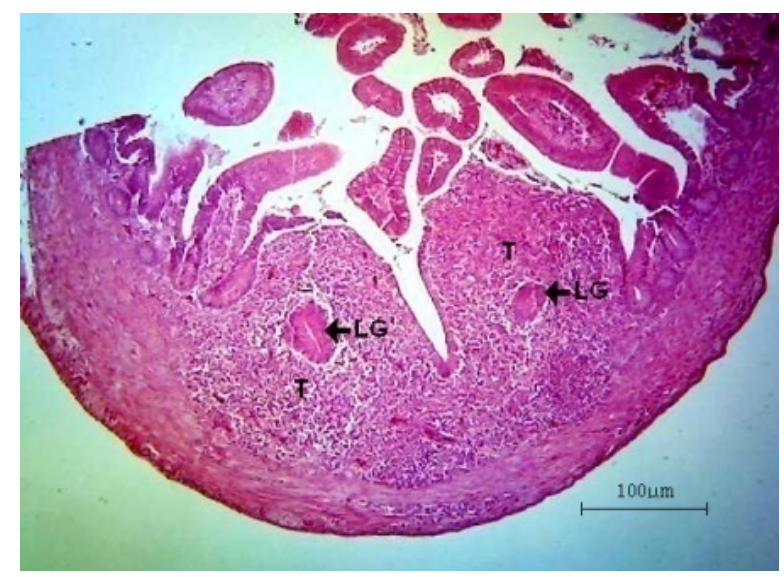

FIGURE 1. Transverse Section of the caecum at day 1 post hatch showing the orientation of the layers of the caecal wall. Note the simple columnar epithelium, EPITH with goblet cells (arrow) of the mucosa and the lymphoid accumulations, LA in the lamina propria- submucosa. M: muscularis; LG: Leiberkuhn gland. X400. 


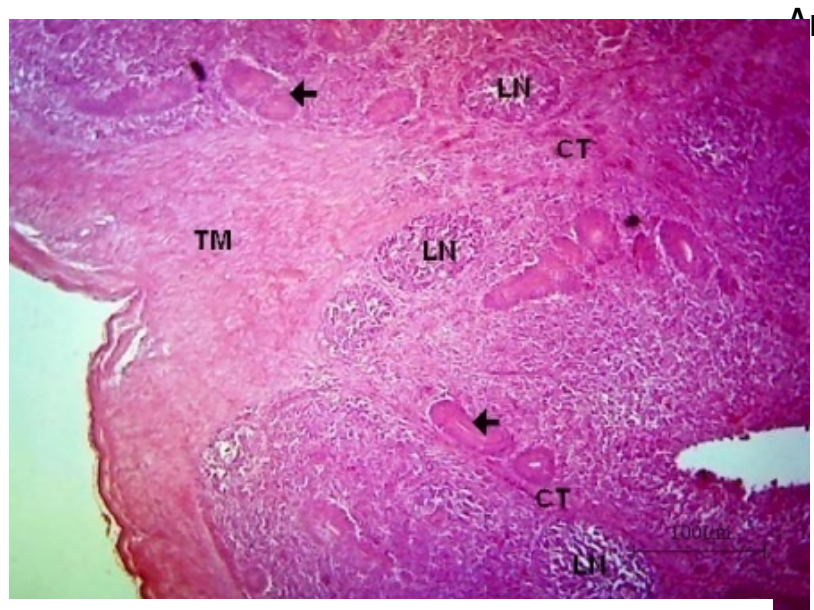

FIGURE 3. Transverse Section of the caecal tonsil at day 28 showing the stroma of the tonsil. Note the lymphatic nodules, LN; Leiberkuhn glands, (arrow) and the intervening connective tissue, CT. TM: tunica muscularis. X100.

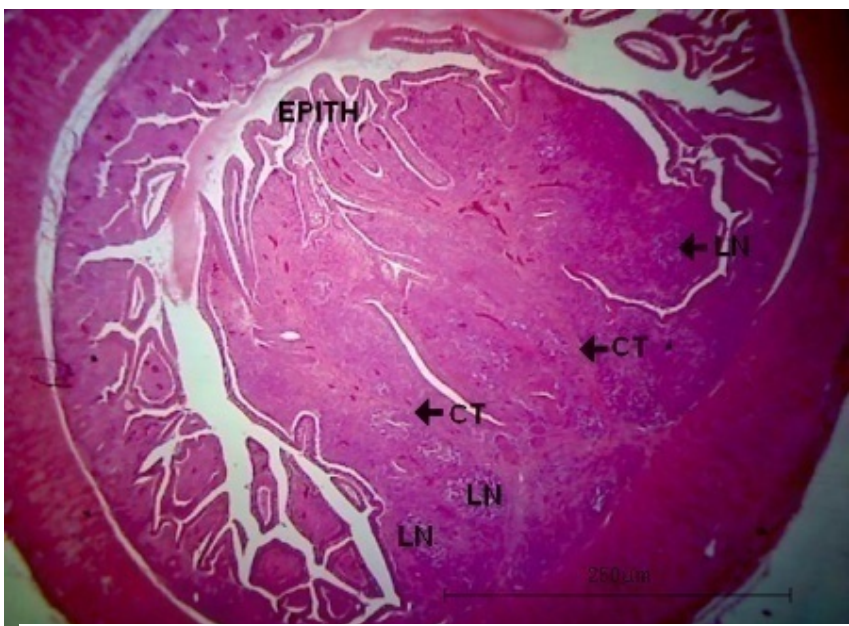

FIGURE 5. Transverse Section of the caecal tonsil at day 84 showing the dimension of the tonsil. Note the epithelium, EPITH; intervening connective tissue, CT; lymphatic nodules, LN. X40.

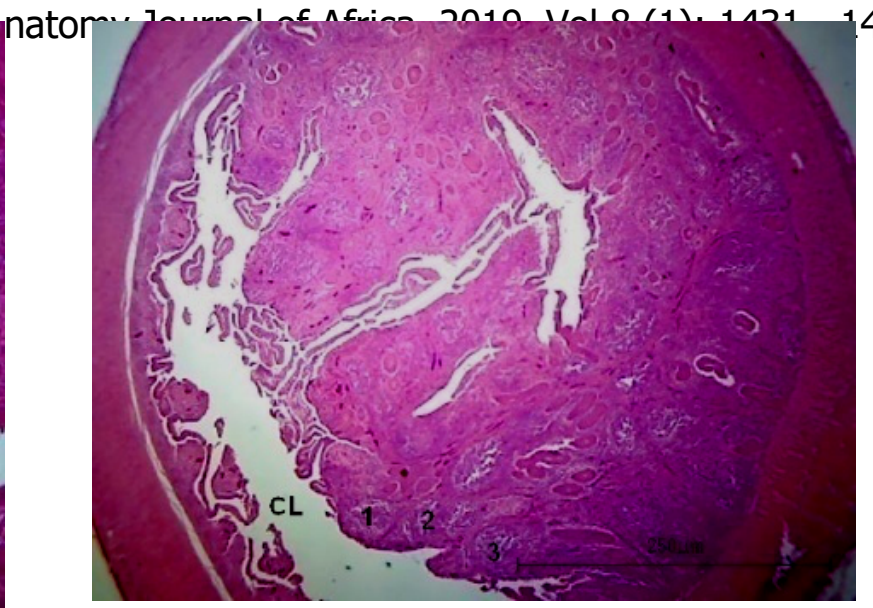

FIGURE 4. Transverse Section of the caecal tonsil at day 56 showing the dimension of the tonsil. Note the proximity of lymphatic nodules, 1 , 2 , and 3 to the mucosa. CL: caecal lumen. X40.

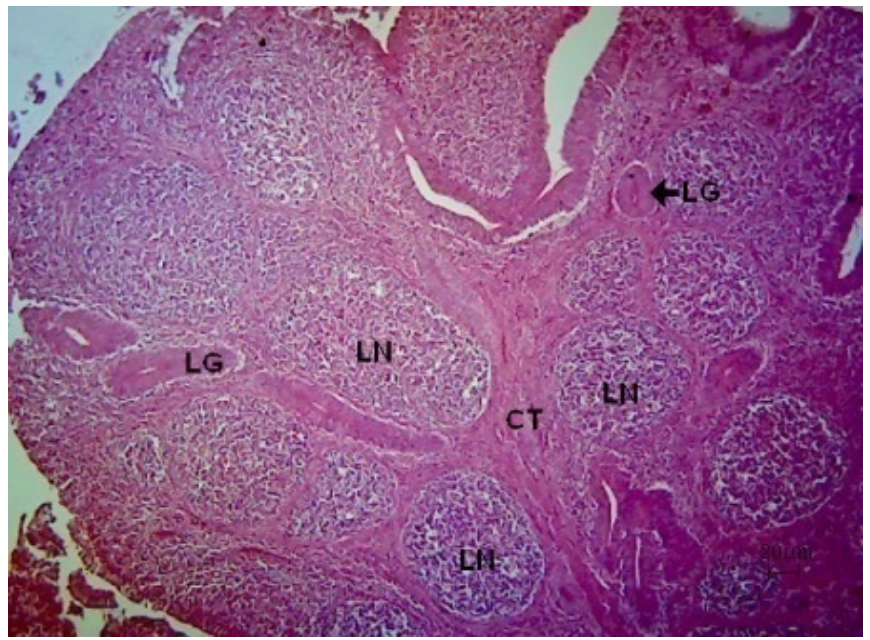

FIGURE 6. Transverse Section of the caecal tonsil at day 112 showing the stroma of the tonsil. Note the numerous lymphatic nodules, $\mathrm{LN}$ and the intervening connective tissue septa, CT. X100 


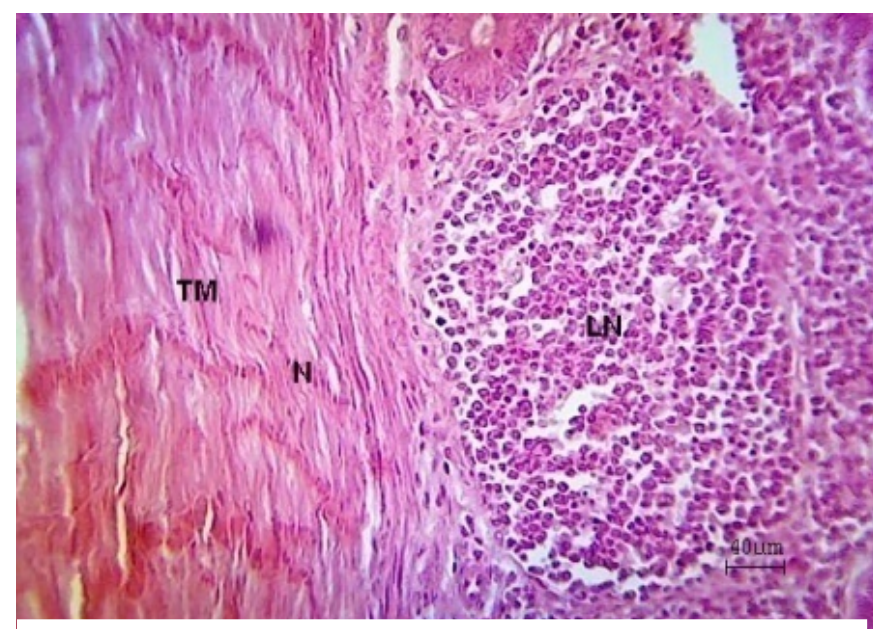

FIGURE 7. Transverse Section of the caecal tonsil at day 126 showing the muscular layer, TM of the caecal wall and a lymphatic nodule, LN. Note the tuft of tissue, $\mathrm{T}$ between the muscularis and nodular capsule, $\mathrm{C} . \mathrm{N}$ : nuclei of muscle cells $\mathrm{X} 400$.

In turkey, the caecal tonsils appeared as ampullaceous structures on the surface of the proximal part of the base of the caecum as observed in the chicken (Rezaian and Hamedi, 2007). In the duck, the closest relative of the galliformes, Kitamura et al. (1976) reported that no such elevated structures indicative of caecal tonsils existed on the surface of the caecal base, but rather the lymphatic tissues were distributed more densely in the proximal one-tenth of the body and the blind sac of the caecum.

The mucosa of the tonsil in turkey comprised simple columnar epithelium with goblet cells and the distribution of both diffused lymphatic tissues and lymphatic nodules were observed in the lamina propria-submucosa. Similar findings were made in the chicken (Akter et al.; 2006; Rezaian and Hamedi, 2007; Majeed et al.; 2009), but Hoffmann-Fezer (1973) reported that the diffused lymphatic tissue with germinal centers occupy only the lamina propria in the chicken. Kitamura et al. (1976) stated that in the duck caecum, the diffused lymphatic tissue and the lymphatic nodule with germinal centers were found to be separately located in the lamina propria and submucosa respectively.

The muscularis of the caecal tonsil in turkey comprised smooth muscle fibers devoid of

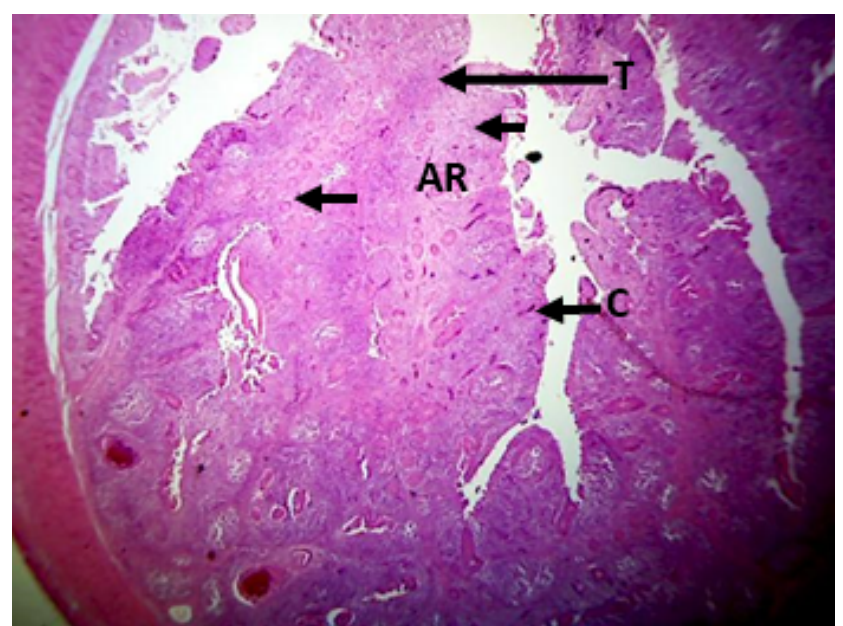

FIGURE 8. Transverse Section of the caecal tonsil at day 140 . Note the extensiveness of the tonsil and the nature of the lymphatic nodules (arrow) in the apical region, $A R$ of the tonsil. $X 40$.

\section{DISCUSSION}

lymphoid accumulation, but in the duck and goose, Georgescu et al. (2007) reported the presence of intramuscular lymphatic nodules in the muscle layer of the caecal tonsil.

The minute aggregates of lymphocytes in the lamina propria-submucosa of the caecum as observed at day 1 probably formed the basal level of lymphocyte accumulation of the caecal tonsil which, Dunon et al. (1997), suggested may coincide with the early reported waves of $\mathrm{T}$ emigration from the thymus. By day 7, there was obvious proliferation of lymphocytes in the tonsil. Befus et al. (1980) reported similar occurrence in the Peyer's patches and caecal tonsil in chicken. The increase in density of lymphoid accumulation probably resulted due to exposure of the birds to the microbial environment, since chicks after clearing their shell started pecking and learned to associate pecking with ingestion and feeding (Hogan, 1984); and increase of lymphoid function in the chick intestines depends on the presence of bacteria and coincides with enterocyte and villus development (Bar-shira et al., 2005). This therefore, implies that after hatching rapid development of the caecal tonsils should be expected since the caecum is exposed to constant invasion by microbial and nonmicrobial antigens of extra-caecal origin as it 
receives the back flowing urine from the urodeum of cloaca through the rectum (Rezaian and Hamedi, 2007). It was reported that in chicken and quail reared in a germ-free environment no germinal centers were evident in the caecal tonsils and lymphoid tissue was remarkably reduced which suggested that the gut flora is essential to stimulate full development of the tonsils (Hedge et al., 1982). Bar-shira and Friedman (1999) reported that the development of lymphoid follicle (nodule) is the hallmark of developing adaptive immune response. These follicles contain naive $T$ and $B$ cells undergoing differentiation and division in the process of generating effectors and memory cells, both of which may migrate to the tissues. The early development of these follicles in the caecal tonsils in turkey emphasized the crucial need for a functional immune mechanism in the gut since it is highly prone to both internal and environmental antigenic influences.

In conclusion, the post-hatch development of the caecal tonsil in turkey is apparently rapid and its morphology is quite similar to those of the chicken and other Galliformes such as the guinea fowl.

\section{REFERENCES}

1. Akter, S.H., Khan, M.Z.I., Jahan, M.R., Karim, M.R. and Islam, M.R. (2006). Histomorphological study of the lymphoid tissues of broiler chickens. Bangl. J. Vet. Med. 4 (2): 87-92.

2. Alboghobeish, N. and Mayahi, M. (2003). Developmental study of lymphoid tissue of bursa of Fabricius in local chicken. The $11^{\text {th }}$ sympo Wld Assoc Vet Lab Diag and OIE Semn on Biotech., Nov. 9-13.

3. Bar-Shira E. and Friedman, A. (1999). Ontogeny of gut associated immune competence in the chick. Israel J. Vet Med.

4. Bar-shira E., Sklan, D., Friedman, A. (2005). Impaired immune responses in broiler hatchling hindgut following delayed access to feed. Vet immuno immunopathol 105: 34-45.

5. Befus, A.D., Johnston, N., Leslie, G.A. and Bienenstock, J. (1980). Gut associated lymphoid tissue in chicken. I. morphology, ontogeny, and some functional characteristics of Peyer's Patches. J. Immunol. 125: 2626-32.

6. Ciriaco, E., Pinera, P.P., Diaz-Esnal, B. and Laura, L. (2003). Age-related changes in the avian primary lymphoid organs (thymus and bursa of Fabricius). Microsc. Res. Tech. 62: 482-487.

7. Cortan, R. S.; Kumur, V. and Robbin, S. L. (1998). Robbin's pathogenic basis of disease. $4^{\text {th }}$ Ed. Philadelphia: W. B. Saunder's; p. $163-164$.

8. Dunon, D., Courtois, D., Vainio, O., Six, A., Chen, C.H., Cooper, M.D., Daugy, J.P. and Imhof, B.A. (1997). Ontogeny of the immune system: gamma/delta and alpha/beta T cells migrate from thymus to the periphery in alternating waves. J. Exp Med. 186: 977-88.

9. Erf, G.F. (2004). Cell-mediated immunity in poultry. Poult. Sci. 83: 580-590.

10. Erf, G.F. (2007). Avian immune system in infectious bursal disease and its role in immunosuppression. Watt Poult. USA Webinar.

11. Fred, D., Bernd, K. and Karel, A. S. (2008). Avian Immunology. Academic Press- Elsevier.

12. Firth, G.A. (1977). The normal lymphatic system of the domestic fowl. Vet. Bull 47: 167-178.

13. Georgescu, B.; Emilia, C.; Predoi, G.; Cornila, N. (2007). Research concerning histostructure of cecal tonsils in some species of domestic birds. Hucrari Stintifice Medicina Veterinara. Vol, $\mathrm{XL}$, Timisoara.

14. Getty, R.(1975). Sisson and Grossman's The Anatomy of the Domestic Animals. 5th. W. B. Saunders Company. Philadelphia. 
15. Hedge, S.N., Rolls, B.A., Turvey, A. and Coates, M.E. (1982). Influence of gut microflora on the lymphoid tissue in chicken (Gallus domesticus) and Japanese quail (Coturnix coturnix Japonica) Comp. Biochem. Physiol. 72A: 205-209.

16. Hoffman-Fezer, G. (1973). Histologische untersuchungen an lymphatischen organic des Huhne (Gallus domesticus) wahrend des ersten lebensjahres. Zeitschrift fur Zellforschung un mikroskopische Anatomic 133: 123-210.

17. Hogan, J.A. (1984). Pecking and Feeding in chicks. Learn motiv. 15: 360-76.

18. Islam, M.N., Khan, M.Z.I., Jahan, M.R., Fujinaga, R. Yanai, A., Kokubu, K and Shinoda, K. (2012). Histomorphological study on prenatal development of the lymphoid organs of native chicken of Bangladesh. Pakistan Vet. J., 32 (2): 175-178.

19. Kitamura, H.; Sugimura, M.; Hashimoto, Y.; Yamano, S. and Kudo, N. (1976). Distribution of lymphatic tissues in Duck Ceca. Jap. J. Vet. Res., 24(1-20):37-42.

20. Majeed, M. F.; Ai-Asadi, F. S.; Al. Nassir, A. N. and Rahi, E. H. (2009). The morphological and histological studies of the cecum in broiler chicken. Bas. J. Vet. Res. Vol.8. No.1.

21. Ratcliffe, M.J.H. (1989). Development of the avian B-lymphocytes lineage. CRC. Crit. Rev. Poult. Biol. 2: 207-234.

22. Razaian, M. and Hamedi, S. (2007). Histological study of the cecal tonsil in the cecum of 4-6 month of age white leghorn chicks. Am. J. Ani. Vet. Sci. 2(2): 50-54. 